\title{
CURVATURE FLOW OF COMPLETE HYPERSURFACES IN HYPERBOLIC SPACE
}

\author{
LING XIAO
}

\begin{abstract}
In this paper we continue our study of finding the curvature flow of complete hypersurfaces in hyperbolic space with a prescribed asymptotic boundary at infinity. Our main results are proved by deriving a priori global gradient estimates and $C^{2}$ estimates.
\end{abstract}

\section{INTRODUCTION}

In this paper, we continue our study of the modified curvature flow problem in hyperbolic space $\mathbb{H}^{n+1}$. Consider a complete Weingarten hypersurface in $\mathbb{H}^{n+1}$ with a prescribed asymptotic boundary $\Gamma$ at infinity, whose principal curvatures satisfy $f\left(\kappa\left[\Sigma_{0}\right]\right) \leq \sigma$ (e.g. we can choose a constant mean curvature graph as found in [NS96]), and is given by an embedding $\mathbf{X}(0): \Omega \rightarrow \mathbb{H}^{n+1}$, where $\Omega \subset \partial_{\infty} \mathbb{H}^{n+1}$. We consider the evolution of such an embedding to produce a family of embeddings $\mathbf{X}: \Omega \times[0, T) \rightarrow \mathbb{H}^{n+1}$ satisfying the following equations

$$
\left\{\begin{array}{lr}
\dot{\mathbf{X}}=(f(\kappa[\Sigma])-\sigma) \nu_{H} & \text { in } \Omega \times[0, T), \\
\mathbf{X}=\Gamma & \text { on } \partial \Omega \times[0, T), \\
\mathbf{X}(0)=\Sigma_{0} & \text { in } \Omega \times\{0\},
\end{array}\right.
$$

where $\kappa[\Sigma(t)]=\left(\kappa_{1}, \cdots, \kappa_{n}\right)$ denotes the hyperbolic principal curvatures of $\Sigma(t)$, $\sigma \in(0,1)$ is a constant, and $\nu_{H}$ denotes the outward unit normal of $\Sigma(t)$ with respect to the hyperbolic metric.

In this paper we shall use the half-space model

$$
\mathbb{H}^{n+1}=\left\{\left(x, x_{n+1}\right) \in \mathbb{R}^{n+1}: x_{n+1}>0\right\}
$$

equipped with the hyperbolic metric

$$
d s^{2}=\frac{\sum_{i=1}^{n+1} d x_{i}^{2}}{x_{n+1}^{2}} .
$$

One identifies the hyperplane $\left\{x_{n+1}=0\right\}=\mathbb{R}^{n} \times\{0\} \subset \mathbb{R}^{n+1}$ as the infinity of $\mathbb{H}^{n+1}$, denoted by $\partial_{\infty} \mathbb{H}^{n+1}$. For convenience we say $\Sigma$ has compact asymptotic boundary if $\partial \Sigma \subset \partial_{\infty} \mathbb{H}^{n+1}$ is compact with respect to the Euclidean metric in $\mathbb{R}^{n}$.

2010 Mathematics Subject Classification. Primary 53C44; Secondary 35K20, 58J35. 
We assume the function $f$ satisfies the following fundamental structure conditions:

$$
f_{i}(\lambda) \equiv \frac{\partial f(\lambda)}{\partial \lambda_{i}}>0 \text { in } K, \quad 1 \leq i \leq n,
$$

$f$ is a concave function in $K$,

and

$$
f>0 \text { in } K, f=0 \text { on } \partial K,
$$

where $K \subset \mathbb{R}^{n}$ is an open symmetric convex cone such that

$$
K_{n}^{+}:=\left\{\lambda \in \mathbb{R}^{n} \text { : each component } \lambda_{i}>0\right\} \subset K .
$$

In addition, we shall assume that $f$ is normalized

$$
f(1, \cdots, 1)=1
$$

and satisfies the more technical assumptions

$$
f \text { is homogeneous of degree one }
$$

and

$$
\lim _{R \rightarrow+\infty} f\left(\lambda_{1}, \cdots, \lambda_{n-1}, \lambda_{n}+R\right) \geq 1+\epsilon_{0} \quad \text { uniformly in } B_{\delta_{0}}(\mathbf{1})
$$

for some fixed $\epsilon_{0}>0$ and $\delta_{0}>0$, where $B_{\delta_{0}}(\mathbf{1})$ is the ball centered at $\mathbf{1}=$ $(1, \cdots, 1) \in \mathbb{R}^{n}$.

As we can see in GS08, an example of a function satisfying all of these assumptions above is given by $f=\left(H_{k} / H_{l}\right)^{\frac{1}{k-l}}, 0 \leq l<k$, defined in $K$, where $H_{l}$ is the normalized $l$-th elementary symmetric polynomial.(e.g, $H_{0}=1, H_{1}=H, H_{n}=K$ the extrinsic Gauss curvature.)

Since $f$ is symmetric, from (1.4), (1.7) and (1.8) we have

$$
f(\lambda) \leq f(\mathbf{1})+\sum f_{i}(\mathbf{1})\left(\lambda_{i}-1\right)=\sum f_{i}(\mathbf{1}) \lambda_{i}=\frac{1}{n} \sum \lambda_{i} \text { in } K
$$

and

$$
\sum f_{i}(\lambda)=f(\lambda)+\sum f_{i}(\lambda)\left(1-\lambda_{i}\right) \geq f(\mathbf{1})=1 \text { in } K .
$$

In this paper, we always assume the initial surface $\Sigma_{0}$ to be connected and orientable, $\Sigma(t)=\left\{(x, u(x, t)) \mid x \in \Omega, x_{n+1}=u, 0 \leq t<T\right\}$ to be the flowing surfaces, and the position vector $\mathbf{X}=(x, u(x, t))$ satisfies the flow equation (1.1). If $\Sigma$ is a complete hypersurface in $\mathbb{H}^{n+1}$ with compact asymptotic boundary at infinity, then the normal vector field of $\Sigma$ is always chosen to be the one pointing to the unique unbounded region in $\mathbb{R}_{+}^{n+1} / \Sigma$. In this case, both the Euclidean and hyperbolic principal curvature of $\Sigma$ are calculated with respect to this normal field.

We shall take $\Gamma=\partial \Omega$, where $\Omega \subset \mathbb{R}^{n}$ is a smooth domain and let $\Gamma_{\epsilon}$ denote its vertical lift. We seek a family of hypersurfaces $\Sigma(t)$ as the graph of a function 
$u(x, t)$ with boundary $\Gamma$ satisfying equation (1.1). Then the coordinate vector fields and upper unit normal are given by

$$
\mathbf{X}_{i}=e_{i}+u_{i} e_{n+1}, \quad \nu_{H}=u \nu=u \frac{-u_{i} e_{i}+e_{n+1}}{w},
$$

where through out this paper $w=\sqrt{1+|\nabla u|^{2}}$ and $e_{n+1}$ is the unit vector in the positive $x_{n+1}$ direction in $\mathbb{R}^{n+1}$.

Notice that

$$
\left\langle\dot{\mathbf{X}}, \nu_{H}\right\rangle_{H}=f-\sigma
$$

which is equivalent to

$$
\left\langle\frac{\partial}{\partial t}(x, u(x, t)), \nu_{H}\right\rangle_{H}=f-\sigma .
$$

Thus the height function $u$ satisfies equation

$$
u_{t}=(F-\sigma) u w .
$$

Therefore problem (1.1) can be represented as the Dirichlet problem for a fully nonlinear second order parabolic equation

$$
\left\{\begin{array}{lr}
u_{t}=u w(f-\sigma) & \text { in } \Omega \times[0, T), \\
u(x, t)=0 & \text { on } \partial \Omega \times[0, T), \\
u(x, 0)=u_{0} & \text { in } \Omega \times\{0\} .
\end{array}\right.
$$

In this paper, we shall focus on proving the long time existence of the modified general curvature flow (MGCF) of a complete embeded hypersurface with initial surface $\Sigma_{0}=\left\{\left(x, u_{0}(x)\right), x \in \Omega\right\}$ satisfying $f\left(\kappa\left[\Sigma_{0}\right]\right) \leq \sigma$ and $1 / w\left(u_{0}\right)>\sigma$. These additional assumptions will be needed in the proof of Proposition 4.3. (Note that for constant mean curvature graph the latter assumption is trivial.) Following the literature we define the class of admissible functions:

$$
\mathcal{A}\left(\Omega_{T}\right)=\left\{u \in C^{2,1}\left(\Omega_{T}\right): \kappa[u] \in K\right\} .
$$

Condition (1.3) implies that equation (1.13) is parabolic for admissible solutions. Our goal is to show that the Dirichlet problem (1.13) admits smooth admissible solutions for all $0<\sigma<1$. Due to the special nature of the problem we saw in GS08, there are substantial technical difficulties to overcome and we have not yet succeeded in finding the solutions for all $\sigma \in(0,1)$. However, we succeed in improving the result in GS08.

Theorem 1.1. Let $\Gamma=\partial \Omega \times\{0\} \subset \mathbb{R}^{n+1}$ where $\Omega$ is a bounded smooth domain in $\mathbb{R}^{n}$. Suppose that the Euclidean mean curvature $\mathcal{H}_{\partial \Omega} \geq 0$ and $\sigma \in(0,1)$ satisfies $\sigma>\sigma_{0}$, where $\sigma_{0}$ is the unique zero in $(0,1)$ of

$$
\phi(a):=\frac{4}{3} a-\frac{1}{27} a^{3}-\frac{1}{27}\left(a^{2}+3\right)^{3 / 2} .
$$

(Numerical calculations show $0.14596<\sigma_{0}<0.14597$.) 
Under conditions (1.3)-(1.9), there exists a solution $\Sigma(t), t \in[0, \infty)$, to the $M G C F$ (1.1) with uniformly bounded principal curvatures

$$
|\kappa[\Sigma(t)]| \leq C \text { on } \Sigma(t), \forall t \in[0, \infty) .
$$

Moreover, $\Sigma(t)=\{(x, u(x, t)) \mid(x, t) \in \Omega \times[0, \infty)\}$ is the flowing surfaces of the unique admissible solution $u(x, t) \in C^{\infty}(\Omega \times(0, \infty)) \cap W_{p}^{2,1}(\Omega \times[0, \infty))$ of the Dirichlet problem (1.13), where $p>4$. Furthermore, for any fixed $t \in[0, \infty)$, $u^{2}(x, t) \in C^{\infty}(\Omega) \cap C^{1+1}(\bar{\Omega})$ and

$$
\begin{gathered}
\sqrt{1+|D u|^{2}} \leq C \text { in } \Omega, \\
u\left|D^{2} u\right| \leq C \text { in } \Omega .
\end{gathered}
$$

In addition, as $t \rightarrow \infty, u(x, t)$ converges uniformly to a function $\tilde{u}(x) \in C^{\infty}(\Omega) \cap$ $C^{1}(\bar{\Omega})$ such that $\Sigma_{\infty}=\{(x, \tilde{u}(x)) \mid x \in \Omega\}$ is a unique complete surface satisfies $f\left(\kappa\left[\Sigma_{\infty}\right]\right)=\sigma$ in $\mathbb{H}^{n+1}$.

Equation (1.13) is degenerate when $u=0$. It is therefore very natural to approximate the boundary condition $u=0$ on $\partial \Omega \times[0, T)$ by $u=\epsilon$ on $\partial \Omega \times[0, T)$, for $\epsilon>0$ sufficiently small. So the problem becomes

$$
\left\{\begin{array}{lr}
u_{t}=u w(f-\sigma) & \text { in } \Omega \times[0, T), \\
u(x, t)=\epsilon & \text { on } \partial \Omega \times[0, T), \\
u(x, 0)=u_{0}^{\epsilon}=u_{0}+\epsilon & \text { in } \Omega \times\{0\},
\end{array}\right.
$$

where $\Sigma_{0}^{\epsilon}=\left\{\left(x, u_{0}^{\epsilon}\right) \mid x \in \Omega\right\}$ satisfies $f\left(\kappa\left[\Sigma_{0}^{\epsilon}\right]\right) \leq \sigma$ and $\frac{1}{w\left(u_{0}^{\epsilon}\right)}>\sigma, \forall x \in \Omega$.

Theorem 1.2. Let $\Omega$ be a bounded smooth domain in $\mathbb{R}^{n}$ with $\mathcal{H}_{\partial \Omega} \geq 0$ and suppose $f$ satisfies (1.3)-(1.9). Then for any $\sigma \in(0,1)$ and $\epsilon>0$ sufficiently small, there exists a unique admissible solution $u^{\epsilon} \in C^{\infty}(\bar{\Omega} \times(0, \infty))$ of the Dirichlet Problem (1.18). Moreover, $u^{\epsilon}$ satisfies the a priori estimates

$$
\begin{aligned}
& \sqrt{1+\left|D u^{\epsilon}\right|^{2}} \leq C \text { in } \Omega \times[0, \infty), \\
& u^{\epsilon}\left|D^{2} u^{\epsilon}\right| \leq C \text { on } \partial \Omega \times[0, \infty),
\end{aligned}
$$

and

$$
u^{\epsilon}\left|D^{2} u^{\epsilon}\right| \leq C(\epsilon, t) \text { in } \Omega \times[0, \infty) .
$$

In particular, $C(\epsilon, t)$ depends exponentially on time $t$.

Remark 1.3. The a priori estimates (1.19) and (1.20) will be proved in section 4 and 5, while (1.21) can be derived by combining Theorem 3.1 and Lemma 6.2 with the standard maximum principle for parabolic equations.

The paper is organized as follows. In Section 2 we establish some basic identities for hypersurface in $\mathbb{H}^{n+1}$. In Section 3 we state the short time existence theorem and derive evolution equations for some geometric quantities. In Section 4 we use the 
mean convex condition on the boundary to establish a sharp global gradient bound for $u$. In Section 5 we show the boundary second derivative estimates. In Section 6 we prove a maximum principle for the maximal hyperbolic principal curvature by using radial graphs (this idea is from GSZ09]). Finally in Section 7 we prove that as $t \rightarrow \infty, \Sigma(t)$ converges uniformly to a hypersurface $\tilde{\Sigma}$ satisfies $f(\kappa[\tilde{\Sigma}])=\sigma$.

\section{Formulas FOR hyPERBolic PRINCIPAL CURVATURES}

2.1. Formulas on hypersurfaces. We will compare the induced hyperbolic and Euclidean metrics and derive some basic identities on a hypersurface.

Let $\Sigma$ be a hypersurface in $\mathbb{H}^{n+1}$. We shall use $g$ and $\nabla$ to denote the induced hyperbolic metric and Levi-Civita connections on $\Sigma$, respectively. Since $\Sigma$ also can be viewed as a submanifold of $\mathbb{R}^{n+1}$, we shall usually identify a geodesic quantity with respect to the Euclidean metric by adding a 'tilde' over the corresponding hyperbolic quantity. For instance, $\tilde{g}$ denotes the induced metric on $\Sigma$ from $\mathbb{R}^{n+1}$, and $\tilde{\nabla}$ is its Levi-Civita connection.

Let $\left(z_{1}, \cdots, z_{n}\right)$ be local coordinates and

$$
\tau_{i}=\frac{\partial}{\partial z_{i}}, \quad i=1, \cdots, n \text {. }
$$

The hyperbolic and Euclidean metrics of $\Sigma$ are given by

$$
g_{i j}=\left\langle\tau_{i}, \tau_{j}\right\rangle_{H}, \quad \tilde{g}_{i j}=\tau_{i} \cdot \tau_{j}=u^{2} g_{i j},
$$

while the second fundamental forms are

$$
\begin{aligned}
& h_{i j}=\left\langle D_{\tau_{i}} \tau_{j}, \nu_{H}\right\rangle_{H}=-\left\langle D_{\tau_{i}} \nu_{H}, \tau_{j}\right\rangle_{H}, \\
& \tilde{h}_{i j}=\nu \cdot \tilde{D}_{\tau_{i}} \tau_{j}=-\tau_{j} \cdot \tilde{D}_{\tau_{i}} \nu
\end{aligned}
$$

where $D$ and $\tilde{D}$ denote the Levi-Civita connection of $\mathbb{H}^{n+1}$ and $\mathbb{R}^{n+1}$, respectively. The following relations are well known (see equation(1.7),(1.8) of [GS08] ):

$$
\begin{gathered}
h_{i j}=\frac{1}{u} \tilde{h}_{i j}+\frac{\nu^{n+1}}{u^{2}} \tilde{g}_{i j} . \\
\kappa_{i}=u \tilde{\kappa}_{i}+\nu^{n+1}, \quad i=1, \cdots, n,
\end{gathered}
$$

where $\nu^{n+1}=\nu \cdot e_{n+1}$.

The Christoffel symbols are related by formula

$$
\Gamma_{i j}^{k}=\tilde{\Gamma}_{i j}^{k}-\frac{1}{u}\left(u_{i} \delta_{k j}+u_{j} \delta_{i k}-\tilde{g}^{k l} u_{l} \tilde{g}_{i j}\right) .
$$

It follows that for $v \in C^{2}(\Sigma)$

$$
\nabla_{i j} v=v_{i j}-\Gamma_{i j}^{k} v_{k}=\tilde{\nabla}_{i j} v+\frac{1}{u}\left(u_{i} v_{j}+u_{j} v_{i}-\tilde{g}^{k l} u_{k} v_{l} \tilde{g}_{i j}\right)
$$

where and in the sequel (if no additional explanation)

$$
v_{i}=\frac{\partial v}{\partial x_{i}}, v_{i j}=\frac{\partial^{2} v}{\partial x_{i} \partial x_{j}}, \text { etc. }
$$


In particular,

$$
\nabla_{i j} u=\tilde{\nabla}_{i j} u+\frac{2 u_{i} u_{j}}{u}-\frac{1}{u} \tilde{g}^{k l} u_{k} u_{l} \tilde{g}_{i j}
$$

Moreover in $\mathbb{R}^{n+1}$,

$$
\begin{gathered}
\tilde{g}^{k l} u_{k} u_{l}=|\tilde{\nabla} u|^{2}=1-\left(\nu^{n+1}\right)^{2} \\
\tilde{\nabla}_{i j} u=\tilde{h}_{i j} \nu^{n+1} .
\end{gathered}
$$

We note that all formulas listed above still hold for general local frame $\tau_{1}, \cdots, \tau_{n}$. In particular, if $\tau_{1}, \cdots, \tau_{n}$ are orthonormal in the hyperbolic metric, then $g_{i j}=\delta_{i j}$ and $\tilde{g}_{i j}=u^{2} \delta_{i j}$.

We now consider equation (1.1) on $\Sigma$. For $K$ as in section 1 , let $\mathcal{A}$ be the vector space of $n \times n$ matrices and

$$
\mathcal{A}_{K}=\left\{A=\left\{a_{i j}\right\} \in \mathcal{A}: \lambda(A) \in K\right\},
$$

where $\lambda(A)=\left(\lambda_{1}, \cdots, \lambda_{n}\right)$ denotes the eigenvalues of $A$. Let $F$ be the function defined by

$$
F(A)=f(\lambda(A)), \quad A \in \mathcal{A}_{K}
$$

and denote

$$
F^{i j}(A)=\frac{\partial F}{\partial a_{i j}}(A), \quad F^{i j, k l}(A)=\frac{\partial^{2} F}{\partial a_{i j} \partial a_{k l}}(A) .
$$

Since $F(A)$ depends only on the eigenvalues of $A$, if $A$ is symmetric then so is the matrix $\left\{F^{i j}(A)\right\}$. Moreover,

$$
F^{i j}(A)=f_{i} \delta_{i j}
$$

when $A$ is diagonal, and

$$
\begin{gathered}
F^{i j}(A) a_{i j}=\sum f_{i}(\lambda(A)) \lambda_{i}=F(A), \\
F^{i j}(A) a_{i k} a_{j k}=\sum f_{i}(\lambda(A)) \lambda_{i}^{2} .
\end{gathered}
$$

Equation (1.13) can therefore be rewritten in a local frame $\tau_{1}, \cdots, \tau_{n}$ in the form

$$
\left\{\begin{array}{lr}
u_{t}=u w(F(A[\Sigma])-\sigma) & (x, t) \in \Omega \times[0, T), \\
u(x, t)=0 & (x, t) \in \partial \Omega \times[0, T), \\
u(x, 0)=u_{0} & (x, t) \in \Omega \times\{0\},
\end{array}\right.
$$

where $A[\Sigma]=\left\{g^{i k} h_{k j}\right\}$. Let $F^{i j}=F^{i j}(A[\Sigma]), F^{i j, k l}=F^{i j, k l}(A[\Sigma])$. 
2.2. Vertical graphs. Suppose $\Sigma$ is locally represented as the graph of a function $u \in C^{2}(\Omega), u>0$, in a domain $\Omega \subset \mathbb{R}^{n}$ :

$$
\Sigma=\left\{(x, u(x)) \in \mathbb{R}^{n+1}: x \in \Omega\right\} .
$$

In this case we take $\nu$ to be the upward (Euclidean) unit normal vector field to $\Sigma$ :

$$
\nu=\left(-\frac{D u}{w}, \frac{1}{w}\right), w=\sqrt{1+|D u|^{2}} .
$$

The Euclidean metric and second fundamental form of $\Sigma$ are given respectively by

$$
\tilde{g}_{i j}=\delta_{i j}+u_{i} u_{j}
$$

and

$$
\tilde{h}_{i j}=\frac{u_{i j}}{w}
$$

As shown in CNS86, the Euclidean principal curvature $\tilde{\kappa}[\Sigma]$ are the eigenvalues of symmetric matrix $\tilde{A}[u]=\left[\tilde{a}_{i j}\right]$ :

$$
\tilde{a}_{i j}:=\frac{1}{w} \gamma^{i k} u_{k l} \gamma^{l j}
$$

where

$$
\gamma^{i j}=\delta_{i j}-\frac{u_{i} u_{j}}{w(1+w)} .
$$

Note that the matrix $\left\{\gamma^{i j}\right\}$ is invertible with the inverse

$$
\gamma_{i j}=\delta_{i j}+\frac{u_{i} u_{j}}{1+w}
$$

which is the square root of $\left\{\tilde{g}_{i j}\right\}$, i.e., $\gamma_{i k} \gamma_{k j}=\tilde{g}_{i j}$. From (2.4) we see that the hyperbolic principal curvatures $\kappa[u]$ of $\Sigma$ are eigenvalues of the matrix $A[u]=$ $\left\{a_{i j}[u]\right\}$ :

$$
a_{i j}:=\frac{1}{w}\left(\delta_{i j}+u \gamma^{i k} u_{k l} \gamma^{l j}\right) .
$$

When $\Sigma$ is a vertical graph we can also define $F(A[\Sigma])=F(A[u])$.

2.3. Radial graphs. Let $\nabla^{\prime}$ denote the covariant derivative on the standard unit sphere $\mathbb{S}^{n}$ in $\mathbb{R}^{n+1}$ and $y=e_{n+1} \cdot \mathbf{z}$ for $\mathbf{z} \in \mathbb{S}^{n} \subset \mathbb{R}^{n+1}$. Let $\tau_{1}, \cdots, \tau_{n}$ be a local frame of smooth vector fields on the upper hemisphere $\mathbb{S}_{+}^{n}$ and denote $\tau_{i} \cdot \tau_{j}=\sigma_{i j}$.

Suppose that locally $\Sigma$ is a radial graph over the upper hemisphere $\mathbb{S}_{+}^{n} \subset \mathbb{R}^{n+1}$, i.e., it is locally represented as

$$
\mathbf{X}=e^{v} \mathbf{z}, \quad \mathbf{z} \in \mathbb{S}_{+}^{n} \subset \mathbb{R}^{n+1} .
$$

The Euclidean metric, outward unit normal vector and second fundamental form of $\Sigma$ are

$$
\begin{gathered}
\tilde{g}_{i j}=e^{2 v}\left(\sigma_{i j}+\nabla_{i}^{\prime} v \nabla_{j}^{\prime} v\right), \\
\nu=\frac{\mathbf{z}-\nabla^{\prime} v}{w}, w=\left(1+\left|\nabla^{\prime} v\right|^{2}\right)^{1 / 2},
\end{gathered}
$$


and

$$
\tilde{h}_{i j}=\frac{e^{v}}{w}\left(\nabla_{i j}^{\prime} v-\nabla_{i}^{\prime} v \nabla_{j}^{\prime} v-\sigma_{i j}\right)
$$

respectively. Therefore the Euclidean principal curvatures are the eigenvalues of the matrix

$$
\tilde{a}_{i j}=\frac{e^{-v}}{w}\left(\gamma^{i k} \nabla_{k l}^{\prime} v \gamma^{l j}-\sigma_{i j}\right)
$$

where

$$
\gamma^{i j}=\sigma^{i j}-\frac{v^{i} v^{j}}{w(1+w)}
$$

and $v^{i}=\sigma^{i k} \nabla_{k}^{\prime} v$. Note that the height function is $u=y e^{v}$. We see that the hyperbolic principal curvatures are the eigenvalues of matrix $A^{s}[v]=\left\{a_{i j}^{s}[v]\right\}$ :

$$
a_{i j}^{s}[v]:=\frac{1}{w}\left(y \gamma^{i k} \nabla_{k l}^{\prime} v \gamma^{l j}-\mathbf{e} \cdot \nabla^{\prime} v \sigma_{i j}\right) .
$$

In this case we can define $F(A[\Sigma])=F\left(A^{s}[v]\right)$.

\section{Short time existence And Evolution equations}

3.1. Short time existence. In order to prove a global existence for the Dirichlet problem (1.18), we first need a short time existence theorem. Here we shall apply Theorem 3.1 of [LX11] directly. For completeness let's restate the theorem as following:

Theorem 3.1. Let $G\left(D^{2} u, D u, u\right)$ be a nonlinear operator that is smooth with respect to $D^{2} u, D u$ and $u$. Suppose that $G$ is defined for a function $u$ belonging to an open set $\Lambda \subset C^{2}(\Omega)$ and $G$ is elliptic for any $u \in \Lambda$, i.e., $G^{i j}>0$, then the initial value problem

$$
\left\{\begin{array}{lr}
u_{t}=G\left(D^{2} u, D u, u\right) & \text { in } \Omega \times\left[0, T^{*}\right), \\
u(x, 0)=u_{0} & \text { in } \Omega \times\{0\}, \\
u(x, t)=0 & \text { on } \partial \Omega \times\left[0, T^{*}\right),
\end{array}\right.
$$

has a unique smooth solution $u$ when $T^{*}=\epsilon>0$ small enough, except for the corner, where $u_{0} \in \Lambda$ be of class $C^{\infty}(\bar{\Omega})$.

Since Theorem 3.1 was proved in LX11, we omit the proof here.

3.2. Evolution equations for some geometric quantities. For the reader's convenience, we now compute the evolution equations for some affine geometric quantities that were first derived in [LX11]. In this section we shall write $F_{i j}=$ $\tilde{\nabla}_{i j} F, u_{i j}=\tilde{\nabla}_{i j} u, F_{j}^{i}=\tilde{g}^{i l} F_{l j}$, etc.

Lemma 3.2. (Evolution of the metrics). The metric $g_{i j}$ and $\tilde{g}_{i j}$ of $\Sigma(t)$ satisfies the evolution equations 


$$
\dot{g}_{i j}=-2 u^{-2} \tilde{g}_{i j}(F-\sigma) w-2 u^{-1}(F-\sigma) \tilde{h}_{i j},
$$

and

$$
\dot{\tilde{g}}_{i j}=-2(F-\sigma) u \tilde{h}_{i j}
$$

Proof. Since $\tilde{g}_{i j}=\tau_{i} \cdot \tau_{j}$,

$$
\begin{aligned}
& \frac{\partial}{\partial t} \tilde{g}_{i j}=2\left\langle\tilde{D}_{\tau_{i}} \dot{X}, \tilde{D}_{\tau_{j}} X\right\rangle \\
& =2\left\langle\tilde{D}_{\tau_{i}}[(F-\sigma) u \nu], \tau_{j}\right\rangle \\
& =2(F-\sigma) u\left\langle\tilde{D}_{\tau_{i}} \nu, \tau_{j}\right\rangle \\
& =-2(F-\sigma) u \tilde{h}_{i j} .
\end{aligned}
$$

From equation (2.1) we get

$$
\begin{aligned}
\frac{\partial}{\partial t} g_{i j} & =-2 u^{-3} \tilde{g}_{i j} u_{t}+u^{-2} \dot{\tilde{g}}_{i j} \\
& =-2 u^{-3} \tilde{g}_{i j}(F-\sigma) u w-2 u^{-2}(F-\sigma) u \tilde{h}_{i j} \\
& =-2 u^{-2} \tilde{g}_{i j}(F-\sigma) w-2 u^{-1}(F-\sigma) \tilde{h}_{i j} .
\end{aligned}
$$

Lemma 3.3. (Evolution of the normal). The normal vector evolves according to

$$
\dot{\nu}=-\tilde{g}^{i j}[(F-\sigma) u]_{i} \tau_{j},
$$

moreover,

$$
\dot{\nu}^{n+1}=-\tilde{g}^{i j}[(F-\sigma) u]_{i} u_{j} .
$$

Proof. Since $\nu$ is the unit normal vector of $\Sigma$, we have $\dot{\nu} \in T(\Sigma)$. Furthermore, differentiating

$$
\left\langle\nu, \tau_{i}\right\rangle=\left\langle\nu, \tilde{D}_{\tau_{i}} X\right\rangle=0,
$$

with respect to $t$ we deduce

$$
\begin{aligned}
\left\langle\dot{\nu}, \tau_{i}\right\rangle & =-\left\langle\nu, \tilde{D}_{\tau_{i}}[(F-\sigma) u \nu]\right\rangle \\
& =-\left\langle\nu,[(F-\sigma) u]_{i} \nu\right\rangle \\
& =-[(F-\sigma) u]_{i} .
\end{aligned}
$$

So we have

$$
\dot{\nu}=-\tilde{g}^{i j}[(F-\sigma) u]_{i} \tau_{j},
$$

and (3.5) follows directly from

$$
\dot{\nu}^{n+1}=\langle\dot{\nu}, \mathbf{e}\rangle=-\tilde{g}^{i j}[(F-\sigma) u]_{i} u_{j} .
$$


Lemma 3.4. (Evolution of the second fundamental form). The second fundamental form evolves according to

$$
\begin{gathered}
\dot{\tilde{h}}_{i}^{l}=[(F-\sigma) u]_{i}^{l}+u(F-\sigma) \tilde{h}_{i}^{k} \tilde{h}_{k}^{l}, \\
\dot{\tilde{h}}_{i j}=[(F-\sigma) u]_{i j}-u(F-\sigma) \tilde{h}_{i}^{k} \tilde{h}_{k j},
\end{gathered}
$$

and

$$
\begin{aligned}
& \dot{h}_{i j}=\frac{1}{u}\left\{[(F-\sigma) u]_{i j}-u(F-\sigma) \tilde{h}_{i}^{k} \tilde{h}_{k j}\right\}-\frac{\tilde{h}_{i j}}{u} w(F-\sigma) \\
& -\left\{\tilde{g}^{k l}[u(F-\sigma)]_{k} u_{l}\right\} \frac{\tilde{g}_{i j}}{u^{2}}-2 \frac{(F-\sigma) \nu^{n+1}}{u} \tilde{h}_{i j}-2 \frac{\tilde{g}_{i j}}{u^{2}}(F-\sigma) .
\end{aligned}
$$

Proof. Differentiating (3.4) with respect to $\tau_{i}$ we get

$$
\frac{\partial}{\partial t} \nu_{i}=-\tilde{g}^{k l}[(F-\sigma) u]_{k i} \tau_{l}-\tilde{g}^{k l}[(F-\sigma) u]_{k} \tilde{D}_{\tau_{i}} \tau_{l} .
$$

On the other hand, in view of the Weingarten Equation

$$
\nu_{i}=-\tilde{g}^{k l} \tilde{h}_{l i} \tau_{k} \Rightarrow \dot{\nu_{i}}=-\dot{\tilde{h}}_{i}^{k} \tau_{k}-\tilde{h}_{i}^{k} \tilde{D}_{\tau_{k}} \dot{X}
$$

where $\tilde{h}_{i}^{k}=\tilde{g}^{k l} \tilde{h}_{l i}$ is mixed tensor. Multiplying by $\tau_{j}$ we get

$$
-\dot{\tilde{h}}_{i}^{k} \tilde{g}_{k j}-\tilde{h}_{i}^{k}\left\langle\tilde{D}_{\tau_{k}} \dot{X}, \tau_{j}\right\rangle=-\tilde{g}^{k l}[(F-\sigma) u]_{k i} \tilde{g}_{l j} .
$$

Thus

$$
\begin{aligned}
\dot{\tilde{h}}_{i}^{k} \tilde{g}_{k j} & =\tilde{g}^{k l}[(F-\sigma) u]_{k i} \tilde{g}_{l j}-\tilde{h}_{i}^{k} u(F-\sigma)\left\langle\tilde{D}_{\tau_{k}} \nu, \tau_{j}\right\rangle \\
& =[(F-\sigma) u]_{i j}+u(F-\sigma) \tilde{h}_{i}^{k} \tilde{h}_{k j} .
\end{aligned}
$$

Multiplying by $\tilde{g}^{j l}$ we get (3.6).

Moreover, since $\tilde{h}_{i j}=\tilde{h}_{i}^{l} \tilde{g}_{l j}$, by equation (3.3) and (3.6) we have

$$
\begin{aligned}
\dot{\tilde{h}}_{i j} & =\dot{\tilde{h}}_{i}^{l} \tilde{g}_{l j}+\tilde{h}_{i}^{l} \dot{\tilde{g}}_{l j} \\
& =[(F-\sigma) u]_{i}^{l} \tilde{g}_{l j}+u(F-\sigma) \tilde{h}_{i}^{k} \tilde{h}_{k}^{l} \tilde{g}_{l j}+\tilde{h}_{i}^{l}\left[-2(F-\sigma) u \tilde{h}_{l j}\right] \\
& =[(F-\sigma) u]_{i j}-u(F-\sigma) \tilde{h}_{i}^{k} \tilde{h}_{k j} .
\end{aligned}
$$

Finally, by differentiating (2.3) with respect to $t$, we get

$$
\begin{aligned}
& \frac{\partial}{\partial t} h_{i j}=\frac{1}{u} \dot{\tilde{h}}_{i j}-\frac{\tilde{h}_{i j}}{u^{2}} u_{t}+\frac{\tilde{g}_{i j}}{u^{2}} \dot{\nu}^{n+1}+\frac{\nu^{n+1}}{u^{2}} \dot{\tilde{g}}_{i j}-2 \frac{\nu^{n+1} \tilde{g}_{i j}}{u^{3}} u_{t} \\
& =\frac{1}{u}\left\{[(F-\sigma) u]_{i j}-u(F-\sigma) \tilde{h}_{i}^{k} \tilde{h}_{k j}\right\}-\frac{\tilde{h}_{i j}}{u} w(F-\sigma) \\
& +\frac{\tilde{g}_{i j}}{u^{2}}\left\{-\tilde{g}^{k l}[u(F-\sigma)]_{k} u_{l}\right\}+\frac{\nu^{n+1}}{u^{2}}\left[-2(F-\sigma) u \tilde{h}_{i j}\right]-2 \frac{\nu^{n+1} \tilde{g}_{i j}}{u^{3}} u w(F-\sigma) \\
& =\frac{1}{u}\left\{[(F-\sigma) u]_{i j}-u(F-\sigma) \tilde{h}_{i}^{k} \tilde{h}_{k j}\right\}-\frac{\tilde{h}_{i j}}{u} w(F-\sigma) \\
& -\left\{\tilde{g}^{k l}[u(F-\sigma)]_{k} u_{l}\right\} \frac{\tilde{g}_{i j}}{u^{2}}-2 \frac{(F-\sigma) \nu^{n+1}}{u} \tilde{h}_{i j}-2 \frac{\tilde{g}_{i j}}{u^{2}}(F-\sigma) .
\end{aligned}
$$


Lemma 3.5. (Evolution of $F$ ). The term $F$ evolves according to the equation

$$
\begin{aligned}
& F_{t}=\frac{1}{u} F^{i j}[(F-\sigma) u]_{i j}+(F-\sigma)\left[\sum f_{s} \kappa_{s}^{2}-2 \nu^{n+1} F+\left(\nu^{n+1}\right)^{2} \sum f_{s}\right] \\
& +w(F-\sigma)\left(F-\nu^{n+1} \sum f_{s}\right)-[(F-\sigma) u]_{i} u^{i} \sum f_{s} .
\end{aligned}
$$

Proof. We consider $F$ with respect to the mixed tensor $h_{i}^{j}$. From equation (2.3), (3.5), and (3.6) we conclude

$$
\begin{aligned}
& F_{t}=F^{i j}\left(h_{i}^{j}\right)_{t}=F^{i j}\left(u \tilde{h}_{i}^{j}+\nu^{n+1} \delta_{i j}\right)_{t} \\
& =u F^{i j}[(F-\sigma) u]_{i}^{j}+u^{2}(F-\sigma) F^{i j} \tilde{h}_{i}^{k} \tilde{h}_{k}^{j} \\
& +u w(F-\sigma) F^{i j} \tilde{h}_{i}^{j}-[(F-\sigma) u]_{i} u^{i} \sum f_{s} \\
& =\frac{1}{u} F^{i j}[(F-\sigma) u]_{i j}+(F-\sigma)\left[\sum f_{s} \kappa_{s}^{2}-2 \nu^{n+1} F+\left(\nu^{n+1}\right)^{2} \sum f_{s}\right] \\
& +w(F-\sigma)\left(F-\nu^{n+1} \sum f_{s}\right)-[(F-\sigma) u]_{i} u^{i} \sum f_{s} .
\end{aligned}
$$

\section{Gradient estimates}

In this section we will show that the angle between the upward unit normal and $e_{n+1}$ axis is bounded above upon approaching the boundary. We will also prove Proposition 4.3 which gives us a global gradient bound for the solution.

The following lemma is similar to Theorem 3.1 of GS10.

Lemma 4.1. For $\epsilon>0$ sufficiently small,

$$
\frac{\sigma-\nu^{n+1}}{u}<\frac{\sqrt{1-\sigma^{2}}}{r_{1}}+\frac{\epsilon(1+\sigma)}{r_{1}^{2}} \text { on } \partial \Omega \times[0, T),
$$

where $r_{1}$ is the maximal radii of exterior sphere to $\partial \Omega$.

Proof. Applying Theorem 3.1 and letting $T$ be small enough, we first assume $r_{1}<$ $\infty$. For a fixed point $x_{0} \in \Gamma^{\epsilon}$, let $\mathbf{e}_{1}$ be the outward unit normal vector to $\Gamma^{\epsilon}$ at $x_{0}$. Let $B_{1}$ be the ball in $\mathbb{R}^{n+1}$ of radius $R_{1}$ centered at $a=\left(x_{0}+r_{1} \mathbf{e}_{1}, R_{1} \sigma\right)$ where $R_{1}$ satisfies $R_{1}^{2}=r_{1}^{2}+\left(\epsilon-R_{1} \sigma\right)^{2}$.

Note that $B_{1} \cap P(\epsilon)=\left\{x \in \mathbb{R}^{n+1} \mid x_{n+1}=\epsilon\right\}$ is an n-ball of radius $r_{1}$, which is externally tangent to $\Gamma^{\epsilon}$. By Lemma 3.3 of [LX10], we know that $B_{1} \cap \Sigma(t)=\emptyset$ for any $t \in[0, T)$. Hence, at $x_{0}$ we have

$$
\nu^{n+1}>-\frac{u-\sigma R_{1}}{R_{1}} .
$$

By an easy computation we also know that,

$$
R_{1} \geq \frac{r_{1}^{2}}{\sqrt{\left(1-\sigma^{2}\right) r_{1}^{2}}+(1+\sigma) \epsilon} .
$$

Therefore (4.1) is proved. In the case that $r_{1}=\infty$, then in the above argument one can replace $r_{1}$ by any $r>0$ and let $r \rightarrow \infty$. 
Now consider the approximation problem

$$
\left\{\begin{array}{lr}
G\left(D^{2} u, D u, u, u_{t}\right)=\frac{1}{u w} u_{t}-F=-\sigma & \text { in } \Omega_{T}, \\
u(x, t)=\epsilon & \text { on } \partial \Omega_{T}, \\
u(x, 0)=u_{0}+\epsilon & \text { in } \Omega \times\{0\} .
\end{array}\right.
$$

By Lemma 4.1 we obtain a boundary gradient estimate

$$
|D u(x, t)| \leq C \text { on } \partial \Omega_{T} .
$$

Similar to Lemma 5.1 of [LX11, we have

Lemma 4.2. If the initial surface $\Sigma_{0}$ satisfies $f\left(\Sigma_{0}\right) \leq \sigma$, then $f(\Sigma(t)) \leq \sigma, \forall(x, t) \in$ $\Omega \times(0, T)$.

Proof. By Lemma 3.5 we have

$$
\begin{aligned}
& \frac{\partial F}{\partial t}-F^{i j} \nabla_{i j} F \\
& =(F-\sigma)\left[\sum f_{s} \kappa_{s}^{2}-\nu^{n+1} F+\left(\nu^{n+1}\right)^{2} \sum f_{s}+w F-2 \sum f_{s}\right] .
\end{aligned}
$$

Now consider the function $\tilde{F}=e^{-\lambda t}(F-\sigma)$,

$$
\begin{aligned}
& \frac{\partial \tilde{F}}{\partial t}-F^{i j} \nabla_{i j} \tilde{F} \\
& =\tilde{F}\left[\sum f_{s} \kappa_{s}^{2}-\nu^{n+1} F+\left(\nu^{n+1}\right)^{2} \sum f_{s}+w F-2 \sum f_{s}-\lambda\right] .
\end{aligned}
$$

If $\tilde{F}$ achieved its positive maximum at an interior point $\left(x_{0}, t_{0}\right) \in \Omega_{T}$, then at this point we would have

$$
\begin{aligned}
& \frac{\partial \tilde{F}}{\partial t}-F^{i j} \nabla_{i j} \tilde{F} \\
& =\tilde{F}\left[\sum f_{s} \kappa_{s}^{2}-\nu^{n+1} F+\left(\nu^{n+1}\right)^{2} \sum f_{s}+w F-2 \sum f_{s}-\lambda\right] \geq 0 .
\end{aligned}
$$

Choosing $\lambda$ big enough leads to a contradiction.

Next we shall appeal to Theorem 3.1 and show that the linearized operator $\mathfrak{L}$ satisfies the maximum principle. Moreover, we can get a uniform $C^{1}$ estimate for the admissible solution.

Proposition 4.3. Let $u(x, t) \in C^{2,1}\left(\Omega_{T}\right)$ be an admissible solution of equation 4.2). Suppose the initial surface $\Sigma_{0}=\left\{\left(x, u_{0}(x)\right), x \in \Omega\right\}$, satisfies $F\left(\Sigma_{0}\right) \leq \sigma$ and $\frac{1}{w\left(u_{0}(x)\right)}>\sigma$. Moreover, suppose $\mathcal{H}_{\partial \Omega} \geq 0$, then $G_{u} \geq 0, F(\Sigma(t)) \leq \sigma$ and $w \leq \frac{1}{\sigma}$ on $\bar{\Omega}_{T}$.

Proof. From

$$
\begin{aligned}
G_{u} & =\frac{\partial G}{\partial u}=\frac{1}{u}\left(-2 F+\sigma+\frac{1}{w} \sum f_{i}\right) \\
& \geq \frac{1}{u}\left(-2 F+\sigma+\frac{1}{w}\right),
\end{aligned}
$$


and the hypotheses on $\Sigma_{0}$ we can see that $\left.G_{u}\right|_{t=0}>0$. Thus when $t$ close to 0 , the linearized operator $\mathfrak{L}$ satisfies the maximum principle. But $\mathfrak{L} u_{k}=0$ so each derivative $u_{k}$ achieves its maximum on $\partial \Omega_{t^{*}}$, where $0<t^{*}<T$ sufficiently small. In particular, $w$ obtains its maximum on $\partial \Omega_{t^{*}}$. By assumption we know that $w\left(u_{0}\right)<\frac{1}{\sigma}$, so we only need to assume that $w$ achieves its maximum on $\partial \Omega \times\left(0, t^{*}\right)$.

Let $\left(0, t_{0}\right) \in \partial \Omega \times\left(0, t^{*}\right)$ be the point where $w$ assumes its maximum. Choose coordinates $\left(x_{1}, \cdots, x_{n}\right)$ at 0 with $x_{n}$ the inner normal direction for $\partial \Omega$. Then at $\left(0, t_{0}\right)$, we have

$$
\begin{gathered}
u_{\alpha}=0,1 \leq \alpha<n, u_{n}>0, u_{n n} \leq 0, \\
\sum u_{\alpha \alpha}=-u_{n}(n-1) \mathcal{H}_{\partial \Omega} \leq 0 .
\end{gathered}
$$

Moreover, by equation (1.10), the hyperbolic mean curvature of $\operatorname{graph}(u) \geq F$. Therefore by implying Theorem 3.1 we have

$$
\frac{n}{\epsilon}\left(\sigma-\frac{1}{w}\right) \leq \frac{1}{w}\left(\sum_{\alpha<n} u_{\alpha \alpha}+\frac{u_{n n}}{w^{2}}\right) \leq-(n-1) \frac{u_{n}}{w} \mathcal{H}_{\partial \Omega} \leq 0 .
$$

Hence $\frac{1}{w} \geq \sigma$ on $\partial \Omega \times\left(0, t^{*}\right)$.

Applying Lemma 4.2 we know $F \leq \sigma$ for all $t \in[0, T)$. Thus $G_{u} \geq 0$ so $\mathcal{L}$ satisfies the maximum principle. Consequently, the estimates must continue to hold as we increase $t^{*}$ up to $T$.

\section{5. $C^{2}$ Boundary estimates}

In this section, we establish boundary estimates for second spatial derivatives of the admissible solutions to the Dirichlet problem (1.18). According to (2.16) we can rewrite equation (1.18) as follows:

$$
\left\{\begin{array}{lr}
\frac{1}{u w} u_{t}-F\left(\frac{1}{w}\left(\delta_{i j}+u \gamma^{i s} u_{s r} \gamma^{r j}\right)\right)=-\sigma & \text { in } \Omega_{T}, \\
u(x, t)=\epsilon & \text { on } \partial \Omega \times[0, T), \\
u(x, 0)=u_{0}+\epsilon & \text { in } \Omega \times\{0\} .
\end{array}\right.
$$

As before we denote

$$
G\left(D^{2} u, D u, u, u_{t}\right)=\frac{1}{u w} u_{t}-F .
$$

Theorem 5.1. Suppose $f$ satisfies equation(1.3)-(1.9). If $\epsilon$ is sufficiently small,

$$
u\left|D^{2} u\right| \leq C \text { on } \partial \Omega \times[0, T),
$$

where $C$ is independent of $\epsilon$ and $t$.

Before we begin our proof note that

$$
\begin{gathered}
G^{s r}:=\frac{\partial G}{\partial u_{s r}}=-\frac{u}{w} F^{i j} \gamma^{i r} \gamma^{s j}, \\
G^{s r} u_{s r}=-F+\frac{1}{w} \sum F^{i i},
\end{gathered}
$$




$$
\begin{gathered}
G_{u}:=\frac{\partial G}{\partial u}=-\frac{1}{w u^{2}} u_{t}-\frac{1}{w} F^{i j} \gamma^{i k} u_{k l} \gamma^{l j} \\
=-\frac{(F-\sigma)}{u}-F^{i j}\left(\frac{a_{i j}}{u}-\frac{1}{u w} \delta_{i j}\right) \\
=-\frac{2 F}{u}+\frac{\sigma}{u}+\frac{1}{w u} \sum F^{i i}, \\
G^{t}:=\frac{\partial G}{\partial u_{t}}=\frac{1}{u w}, \\
G^{s}:=\frac{\partial G}{\partial u_{s}} \quad \\
=-\frac{u_{t} u_{s}}{u w^{3}}+\frac{u_{s}}{w^{2}} F+\frac{2}{w} F^{i j} a_{i k}\left(\frac{w u_{k} \gamma^{s j}+u_{j} \gamma^{k s}}{1+w}\right)-\frac{2}{w^{2}} F^{i j} u_{i} \gamma^{s j} \\
=-\frac{(F-\sigma)}{w^{2}} u_{s}+\frac{u_{s}}{w^{2}} F+\frac{2}{w} F^{i j} a_{i k}\left(\frac{w u_{k} \gamma^{s j}+u_{j} \gamma^{k s}}{1+w}\right)-\frac{2}{w^{2}} F^{i j} u_{i} \gamma^{s j} \\
=\frac{u_{s}}{w^{2}} \sigma+\frac{2}{w} F^{i j} a_{i k}\left(\frac{w u_{k} \gamma^{s j}+u_{j} \gamma^{k s}}{1+w}\right)-\frac{2}{w^{2}} F^{i j} u_{i} \gamma^{s j} .
\end{gathered}
$$

Thus

$$
G^{s} u_{s}=\frac{w^{2}-1}{w^{2}} \sigma+\frac{2}{w^{2}} F^{i j} a_{i k} u_{k} u_{j}-\frac{2}{w^{3}} F^{i j} u_{i} u_{j}
$$

and

$$
\sum\left|G^{s}\right| \leq \frac{\sigma}{w}+\frac{2}{w}\left(\sum F^{i i}+\sum f_{i}\left|\kappa_{i}\right|\right) .
$$

Now let $\mathcal{L}^{\prime}$ denote the partial linearized operator of $G$ at $u$ :

$$
\mathcal{L}^{\prime}=\mathcal{L}-G_{u}=G^{t} \partial_{t}+G^{s r} \partial_{s} \partial_{r}+G^{s} \partial_{s} .
$$

By equation (5.5), (5.7) and (5.9) we get

$$
\begin{aligned}
\mathcal{L}^{\prime} u & =G^{t} u_{t}+G^{s r} u_{s r}+G^{s} u_{s} \\
& =\frac{1}{u w} u w(F-\sigma)-F+\frac{1}{w} \sum F^{i i}+\left(1-\frac{1}{w^{2}}\right) \sigma \frac{2}{w^{2}} F^{i j} a_{i k} u_{k} u_{j}-\frac{2}{w^{3}} F^{i j} u_{i} u_{j} \\
& =-\frac{1}{w^{2}} \sigma+\frac{1}{w} \sum F^{i i}+\frac{2}{w^{2}} F^{i j} a_{i k} u_{k} u_{j}-\frac{2}{w^{3}} F^{i j} u_{i} u_{j},
\end{aligned}
$$

hence

$$
\begin{aligned}
\mathcal{L} \frac{1}{u} & =G^{t}\left(-\frac{u_{t}}{u^{2}}\right)+G^{s r} \partial_{s}\left(-\frac{u_{r}}{u^{2}}\right)+G^{s}\left(-\frac{u_{s}}{u^{2}}\right)+G_{u}\left(\frac{1}{u}\right) \\
& =-\frac{1}{u^{2}}\left(G^{t} u_{t}+G^{s r} u_{s r}+G^{s} u_{s}\right)+2 G^{s r} \frac{u_{r} u_{s}}{u^{3}}+G_{u} \frac{1}{u} \\
& =-\frac{1}{u^{2}}\left(-\frac{1}{w^{2}} \sigma+\frac{1}{w} \sum F^{i i}+\frac{2}{w^{2}} F^{i j} a_{i k} u_{k} u_{j}-\frac{2}{w^{3}} F^{i j} u_{i} u_{j}\right) \\
& +2 G^{s r} \frac{u_{r} u_{s}}{u^{3}}+\frac{1}{u}\left(-2 \frac{F}{u}+\frac{\sigma}{u}+\frac{1}{u w} \sum F^{i i}\right) \\
& =\frac{1+w^{2}}{u^{2} w^{2}} \sigma-2 \frac{F}{u^{2}}-\frac{2}{u^{2} w^{2}} F^{i j} a_{i k} u_{k} u_{j} .
\end{aligned}
$$


Lemma 5.2. Suppose that $f$ satisfies (1.3), (1.4), (1.7) and (1.8). Then

$$
\mathcal{L}\left(1-\frac{\epsilon}{u}\right) \geq \frac{\epsilon}{u^{2} w}(1-\sigma) \sum f_{i}+\frac{2 \epsilon}{u^{2} w^{2}} F^{i j} a_{i k} u_{k} u_{j} \text { in } \Omega_{T} .
$$

Proof. By equation (5.12) and Proposition 4.3

$$
\begin{aligned}
& \mathcal{L}\left(1-\frac{\epsilon}{u}\right)=\mathcal{L}^{\prime}\left(1-\frac{\epsilon}{u}\right)+G_{u}\left(1-\frac{\epsilon}{u}\right) \\
& \geq \mathcal{L}^{\prime}\left(1-\frac{\epsilon}{u}\right)=-\epsilon \mathcal{L}^{\prime}\left(\frac{1}{u}\right)=-\epsilon\left(\mathcal{L}-G_{u}\right) \frac{1}{u} \\
& \geq \frac{\epsilon}{u^{2} w}(1-\sigma) \sum f_{i}+\frac{2 \epsilon}{u^{2} w^{2}} F^{i j} a_{i k} u_{k} u_{j} .
\end{aligned}
$$

Recall that for symmetric matrix $A=A[u]$ we can uniquely define the symmetric matrices

$$
|A|=\left\{A A^{T}\right\}^{\frac{1}{2}}, A^{+}=\frac{1}{2}(|A|+A), A^{-}=\frac{1}{2}(|A|-A)
$$

which all commute and satisfy $A^{+} A^{-}=0$. Moreover, $F=F^{i j}$ commutes with $|A|$, $A^{ \pm}$so all simultaneously diagonalizable. Write $A^{ \pm}=\left\{a_{i j}^{ \pm}\right\}$and define

$$
L=\mathcal{L}+\frac{2}{w^{2}} F^{i j} a_{i k}^{-} u_{k} \partial_{j} .
$$

Corollary 5.3. Suppose that $f$ satisfies (1.3), (1.4), (1.7) and (1.8). Then

$$
L\left(1-\frac{\epsilon}{u}\right) \geq \frac{\epsilon(1-\sigma)}{u^{2} w} \sum f_{i} .
$$

Finally we need to point out that, similar to CNS84 we can prove

Lemma 5.4. Suppose that $f$ satisfies (1.3), (1.4), 1.7) and (1.8). Then

$$
\mathcal{L}\left(x_{i} u_{j}-x_{j} u_{i}\right)=0, \mathcal{L} u_{i}=0,1 \leq i, j \leq n .
$$

Proof of Theorem 5.1. Consider an arbitrary point on $\partial \Omega$, which we may assume to be the origin of $\mathbb{R}^{n}$, and choose the coordinates so that the positive $x_{n}$ axis is the interior normal to $\partial \Omega$ at the origin. There exists a uniform constant $r>0$ such that $\partial \Omega \cap B_{r}(0)$ can be represented as a graph

$$
x_{n}=\frac{1}{2} \sum_{\alpha, \beta<n} B_{\alpha \beta} x_{\alpha} x_{\beta}+O\left(\left|x^{\prime}\right|^{3}\right)=\rho\left(x^{\prime}\right), \quad x^{\prime}=\left(x_{1}, \cdots, x_{n-1}\right) .
$$

Since $u \equiv \epsilon$, on $\partial \Omega \times[0, T)$, i.e., $u\left(x^{\prime}, \rho\left(x^{\prime}\right)\right) \equiv \epsilon$ for $\forall t \in[0, T)$, we have at the origin that

$$
u_{\alpha}+u_{n} B_{\alpha \beta} x_{\beta}=0, u_{\alpha \beta}+u_{n} \rho_{\alpha \beta}=0, \forall t \in[0, T) \text { and } \alpha, \beta<n \text {. }
$$

As in CNS84, let $T_{\alpha}=\partial_{\alpha}+\sum_{\beta<n} B_{\alpha \beta}\left(x_{\beta} \partial_{n}-x_{n} \partial_{\beta}\right)$. For fixed $\alpha<n$, we have

$$
\begin{gathered}
\left|T_{\alpha} u\right| \leq C \text { in }\left\{\Omega \cap B_{\epsilon}(0)\right\} \times[0, T), \\
\left|T_{\alpha} u\right| \leq C|x|^{2} \text { on }\left\{\partial \Omega \cap B_{\epsilon}(0)\right\} \times[0, T),
\end{gathered}
$$


where $C$ is independent of $\epsilon$ and $T$. Moreover by Lemma 5.4

$$
\mathcal{L} T_{\alpha} u=0 .
$$

Now define

$$
\phi= \pm T_{\alpha} u+\frac{1}{2} \sum_{l<n} u_{l}^{2}-\frac{C}{\epsilon^{2}}|x|^{2},
$$

where $C$ is chosen to be large enough (and independent of $\epsilon$ and $T$ ) so that $\phi \leq 0$ on $\partial\left(\Omega \cap B_{\epsilon}(0)\right) \times[0, T)$. Since $u_{0} \in C^{2}(\Omega)$ is given, from Taylor's theorem we can assume in $\Omega \times B_{\delta}(0), \delta>\epsilon>0$ is small, there exists $a_{1}, b_{1}, b_{2}$ and $c_{1}>0$ so that

$$
u_{0}(x) \geq \epsilon+a_{1} x_{n}, \quad\left|T_{\alpha} u_{0}\right| \leq b_{1} x_{n}+b_{2}|x|^{2} \text { and }\left|u_{0 l}\right| \leq c_{1}|x| .
$$

Therefore, we can choose a constant $C_{1}>0$ such that

$$
\phi-C_{1}\left(1-\frac{\epsilon}{u}\right) \leq 0 \text { on }\left\{\Omega \cap B_{\epsilon}(0)\right\} \times\{0\},
$$

here and in the future, all $C$ and $C_{i}(i=1,2, \cdots)$ denote constants independent of $\epsilon$ and $t$.

\section{Lemma 5.5.}

$$
\mathcal{L} \phi \leq \sum_{l<n} G^{s r} u_{l s} u_{l r}+\frac{C}{\epsilon}\left(\sum f_{i}+\sum f_{i}\left|\kappa_{i}\right|\right) \text { in }\left\{\Omega \cap B_{\epsilon}(0)\right\} \times[0, T) .
$$

Proof. Since

$$
\begin{aligned}
& \mathcal{L}\left(|x|^{2}\right)=G^{s r} \partial_{s} \partial_{r}|x|^{2}+G^{s} \partial_{s}|x|^{2}+G_{u}|x|^{2} \\
& \leq\left.\left|2 \sum G^{s s}+2 \sum x_{s} G^{s}+\right| x\right|^{2} G_{u} \mid \\
& \leq 2\left|\sum G^{s s}\right|+2 \epsilon\left|G^{s}\right|+\epsilon^{2}\left|G_{u}\right| \\
& \leq \frac{2 C \epsilon}{w} \sum f_{i}+2 \epsilon\left(\frac{\sigma}{w}+\frac{2}{w}\left(\sum f_{i}+\sum f_{i}\left|\kappa_{i}\right|\right)\right)+C \epsilon\left(\sum f_{i}+\sum f_{i}\left|\kappa_{i}\right|\right)
\end{aligned}
$$

where we applied lemma 2.1 of GS08] and Lemma 3.3 of [LX10].

Combining (5.23) with Lemma 5.4 we obtain (5.22).

Following Ivochkina, Lin and Trudinger [ILT96] we have

Proposition 5.6. At each point in $\left\{\Omega \cap B_{\epsilon}(0)\right\} \times[0, T)$ there is an index $r$ such that

$$
\sum_{l<n} G^{s r} u_{l s} u_{l r} \leq-c_{0} u \sum_{i \neq r} f_{i} \tilde{\kappa}_{i}^{2} \leq \frac{c_{0}}{2 u}\left(\frac{2}{w^{2}} \sum f_{i}-\sum_{i \neq r} f_{i} \kappa_{i}^{2}\right) .
$$

Proof. Let $P$ be an orthogonal matrix that simultaneously diagonalizes $\left\{F^{i j}\right\}$ and $\tilde{A}=\left\{\tilde{a}_{i j}\right\}=\left\{\frac{1}{w} \gamma^{i k} u_{k l} \gamma^{l j}\right\}$, where $\gamma^{i j}=\delta_{i j}-\frac{u_{i} u_{j}}{w(1+w)}$. 
Note that $w \tilde{a}_{i j} \gamma_{j l}=\gamma^{i k} u_{k l}$ and so we have

$$
\begin{aligned}
& \sum_{l<n} G^{s r} u_{l s} u_{l r}=-u w F^{i j} \tilde{a}_{i q} \tilde{a}_{j p} \gamma_{q l} \gamma_{p l} \\
& =-u w \sum_{l<n} f_{i} \tilde{\kappa}_{i}^{2} P_{p i} \gamma_{p l} P_{q i} \gamma_{q l} \\
& =-u w \sum_{l<n} f_{i} \tilde{\kappa}_{i}^{2} b_{l i}^{2}
\end{aligned}
$$

where $B=\left\{b_{r s}\right\}=\left\{P_{i r} \gamma_{i s}\right\}$ and $\operatorname{det}(B)=\operatorname{det}\left(B^{T}\right)=w$.

Suppose for some $i$, say $i=1$, we have

$$
\sum_{l<n} b_{l 1}^{2}<\theta^{2} .
$$

Expanding $\operatorname{det} B$ by cofactors along the first column gives

$$
1 \leq w=\operatorname{det} B=b_{11} C^{11}+\cdots+b_{n-11} C^{1 n-1}+b_{n 1} \operatorname{det} M \leq c_{1} \theta+c_{2} \operatorname{det} M,
$$

where $c_{1}, c_{2}$ are independent of $\epsilon$ and $T$, and

$$
M=\left[\begin{array}{ccc}
b_{12} & \cdots & b_{n-12} \\
\vdots & \ddots & \vdots \\
b_{1 n} & \cdots & b_{n-1 n}
\end{array}\right]
$$

Therefore, $\operatorname{det} M \geq \frac{1-c_{1} \theta}{c_{2}}$. Now expanding $\operatorname{det} M$ by cofactor along row $r \geq 2$ gives $\operatorname{det} M \leq c_{3}\left(\sum_{l<n} b_{l r}^{2}\right)^{1 / 2}$, where $c_{3}$ is independent of $\epsilon$ and $T$. Hence

$$
\sum_{l<n} b_{l r}^{2} \geq\left(\frac{1-c_{1} \theta}{c_{2} c_{3}}\right)^{2} .
$$

Choosing $\theta<\frac{1}{2 c_{1}}$ we conclude that for some $r$

$$
\sum_{l<n} G^{s r} u_{l s} u_{l r} \leq-c_{0} u \sum_{i \neq r} f_{i} \tilde{\kappa}_{i}^{2} .
$$

Finally (5.24) follows from equation (2.4).

Proposition 5.7. Let $L$ be defined by (5.15). Then

$$
L \phi \leq C_{2}\left(\frac{1}{\epsilon} \sum f_{i}-G^{i j} \phi_{i} \phi_{j}\right)
$$

for a controlled constant $C_{2}$ independent of $\epsilon$ and $t$.

Proof. By Lemma 5.5 and Proposition 5.6.

$$
\begin{aligned}
& L \phi=\mathcal{L} \phi+\frac{2}{w^{2}} F^{i j} a_{i k}^{-} u_{k} \partial_{j} \phi \\
& \leq \sum_{l<n} G^{s r} u_{l s} u_{l r}+\frac{C}{\epsilon}\left(\sum f_{i}+\sum f_{i}\left|\kappa_{i}\right|\right)+\frac{2}{w^{2}} F^{i j} a_{i k}^{-} u_{k} \phi_{j} \\
& \leq \frac{c_{0}}{u w^{2}} \sum f_{i}-\frac{c_{0}}{2 u} \sum_{i \neq r} f_{i} \kappa_{i}^{2}+\frac{C}{\epsilon}\left(\sum f_{i}+\sum f_{i}\left|\kappa_{i}\right|\right)+\frac{2}{w^{2}} F^{i j} a_{i k} u_{k} \phi_{j} .
\end{aligned}
$$


Implying the generalized Schwarz inequality,

$$
\begin{aligned}
\frac{2}{w^{2}}\left|F^{i j} a_{i k}^{-} u_{k} \phi_{j}\right| & \leq 2\left(u F^{i j} \phi_{i} \phi_{j}\right)^{\frac{1}{2}}\left(\frac{1}{u} F^{i j} a_{i l}^{-} a_{k j}^{-} \frac{u_{k} u_{l}}{w^{2}}\right)^{\frac{1}{2}} \\
& \leq \frac{c_{0}}{8 n u} \sum_{\kappa_{i}<0} f_{i} \kappa_{i}^{2}-C G^{i j} \phi_{i} \phi_{j},
\end{aligned}
$$

where we have used Lemma 2.1 of GS08 to compare $u F^{i j} \phi_{i} \phi_{j}$ to $-G^{i j} \phi_{i} \phi_{j}$. Moreover,

$$
\sum f_{i}\left|\kappa_{i}\right|=\sum_{\kappa_{i}>0} f_{i} \kappa_{i}-\sum_{\kappa_{i}<0} f_{i} \kappa_{i}=F+2 \sum_{\kappa_{i}<0} f_{i}\left|\kappa_{i}\right| .
$$

Hence we get equation (5.27) with $C_{2}$ independent of $\epsilon$ and $t$.

Let $h=\left(e^{C_{2} \phi}-1\right)-A\left(1-\frac{\epsilon}{u}\right)$, with $C_{2}$ defined as before and $A$ to be determined later. From equation (5.21) we know that when $A$ is chosen large enough

$$
h \leq 0 \text { on } \partial\left\{\left(\Omega \cap B_{\epsilon}(0)\right) \times[0, T)\right\} .
$$

Moreover, by Proposition 5.7 and Corollary 5.3 we get

$$
L h \leq 0 \text { in }\left(\Omega \cap B_{\epsilon}(0)\right) \times[0, T) .
$$

Therefore by the maximum principle we conclude that $h \leq 0$ in $\left(\Omega \cap B_{\epsilon}(0)\right) \times[0, T)$. Since $h(0, t)=0$, we have that $h_{n}(0, t) \leq 0$ for all $t \in[0, T)$ which gives

$$
\left|u_{\alpha n}(0, t)\right| \leq \frac{A}{C_{2} \epsilon} u_{n}(0, t) \text { for all } t \in[0, T) .
$$

Finally, $\left|u_{n n}(0, t)\right|$ can be estimated as in [L.Xiao] section 6 using the hypothesis (1.9). For completeness we include the argument here. For any $t \in[0, T)$, we may assume $\left[u_{\alpha \beta}(0, t)\right]$ to be diagonal. Note also that $u_{\alpha}(0, t)=0$ for $\alpha<n$. We have at $(x, t)=(0, t)$

$$
A[u]=\frac{1}{w}\left[\begin{array}{cccc}
1+u u_{11} & 0 & \cdots & \frac{u u_{1 n}}{w} \\
0 & 1+u u_{22} & \cdots & \frac{u u_{2 n}}{w} \\
\vdots & \vdots & \ddots & \vdots \\
\frac{u u_{n 1}}{w} & \frac{u u_{n 2}}{w} & \cdots & 1+\frac{u u_{n n}}{w^{2}}
\end{array}\right]
$$

By lemma 1.2 in CNS85, if $\epsilon u_{n n}(0)$ is very large, the eigenvalues $\lambda_{1}, \cdots, \lambda_{n}$ of $A[u]$ are given by

$$
\begin{aligned}
\lambda_{\alpha} & =\frac{1}{w}\left(1+\epsilon u_{\alpha \alpha}(0)\right)+o(1), \alpha<n \\
\lambda_{n} & =\frac{\epsilon u_{n n}(0)}{w^{3}}\left(1+O\left(\frac{1}{\epsilon u_{n n}(0)}\right)\right) .
\end{aligned}
$$

If $\epsilon u_{n n} \geq R$ where $R$ is a uniform constant, then by (1.8), (1.9) and Proposition 4.3 we have

$$
\sigma \geq \frac{1}{w} F(w A[u])(0) \geq(\sigma-C \epsilon)\left(1+\frac{\epsilon_{0}}{2}\right)>\sigma
$$

which is a contradiction. Therefore

$$
\left|u_{n n}(0)\right| \leq \frac{R}{\epsilon}
$$


and the proof is completed.

\section{6. $C^{2}$ Global estimates}

In this section we will prove a maximum principle for the largest hyperbolic principal curvature $\kappa_{\max }(x, t)$ of solutions of $f(\kappa[u(x, t)])=\sigma$.

As before, we denote the metric in $\mathbb{H}^{n+1}$ by $g_{i j}$ and denote the hyperbolic second fundamental form by $h_{i j}$. Now consider function

$$
\varphi=\max _{(x, t) \in \bar{\Omega}_{T}} \frac{\kappa_{\max }(x, t)}{\nu^{n+1}-a},
$$

where $\inf _{\bar{\Omega}_{T}} \nu^{n+1}>a$.

Theorem 6.1. Suppose $f$ satisfies [1.3)-(1.8) and $\sigma \in(0,1)$ satisfies $\sigma>\sigma_{0}$, where $\sigma_{0}$ is the unique zero in $(0,1)$ of

$$
\phi(a):=\frac{4}{3} a-\frac{1}{27} a^{3}-\frac{1}{27}\left(a^{2}+3\right)^{\frac{3}{2}} .
$$

Let $u \in C^{4,2}(\Omega \times[0, T))$ be an admissible solution of (1.18) such that $\nu^{n+1}(x, t)=$ $\frac{1}{w} \geq \sigma$, for all $(x, t) \in \Omega_{T}$. Then at an interior maximum of $\varphi$, there is a constant $C$ (independent of $\epsilon$ and $t$ ), such that

$$
\kappa_{\max } \leq C
$$

Numerical calculations show $0.14596<\sigma_{0}<0.14597$.

We begin the proof of Theorem 6.1 which is long and computational.

Assume $\varphi$ achieves its maximum at an interior point $\left(x_{0}, t_{0}\right)$. We may rewrite $\Sigma\left(t_{0}\right)$ locally near $\mathbf{X}_{0}=\left(x_{0}, u\left(x_{0}, t_{0}\right)\right)$ as a radial graph $\mathbf{X}=e^{v(\mathbf{z}, t)} \mathbf{z}, \quad(\mathbf{z}, t) \in$ $\mathbb{S}_{+}^{n} \times(0, T)$, such that $\nu\left(\mathbf{X}_{0}\right)=\mathbf{z}_{0}$, and we may also choose the local coordinates around $\mathbf{z}_{0} \in \mathbb{S}_{+}^{n}$ such that at $\left(\mathbf{z}_{0}, t_{0}\right)$

$$
\tilde{g}_{i j}=\delta_{i j} \text { and } \frac{\partial \tilde{g}_{i j}}{\partial \theta^{k}}=0 .
$$

By a standard calculation, we also know that $v(\mathbf{z}, t)$ satisfies

$$
v_{t}=y w(f-\sigma) .
$$

Moreover, we can also assume $\tilde{h}_{i j}$ is diagnalized at $\left(\mathbf{z}_{0}, t_{0}\right)$. At last, since dilation is an isometry for radial graph, without loss of generality we may assume $v\left(\mathbf{z}_{0}, t_{0}\right)=0$ Therefore at $\left(\mathbf{z}_{0}, t_{0}\right)$ we have

$$
\begin{gathered}
g_{i j}=\frac{\tilde{g}_{i j}}{u^{2}}=\frac{\delta_{i j}}{y^{2}} \text { and } g^{i j}=u^{2} \tilde{g}^{i j}=y^{2} \delta_{i j}, \\
h_{i j}=\frac{1}{u} \tilde{h}_{i j}+\frac{\nu^{n+1}}{u^{2}} \tilde{g}_{i j}=\frac{\tilde{h}_{i j}}{y}+\frac{\delta_{i j}}{y} .
\end{gathered}
$$


Differentiating (2.18) with respect to $\theta^{k}$ we get

$$
\begin{aligned}
& \frac{\partial \tilde{g}_{i j}}{\partial \theta^{k}}=\frac{\partial\left[e^{2 v}\left(\sigma_{i j}+v_{i} v_{j}\right)\right]}{\partial \theta^{k}} \\
& =2 e^{2 v} v_{k}\left(\sigma_{i j}+v_{i} v_{j}\right)+e^{2 v}\left(\frac{\partial \sigma_{i j}}{\partial \theta^{k}}+v_{i k} v_{j}+v_{i} v_{j k}\right)=0 .
\end{aligned}
$$

Since $\nu\left(\mathbf{X}_{0}\right)=\mathbf{z}_{0}$, we conclude that at $\left(\mathbf{z}_{0}, t_{0}\right)$

$$
\frac{\partial \sigma_{i j}}{\partial \theta^{k}}=0
$$

which implies

$$
\Gamma_{i j}^{\prime k}=0
$$

Thus

$$
\nabla_{i j}^{\prime} v=v_{i j}=\tilde{\nabla}_{i j} v
$$

where $\tilde{\nabla}_{i j}$ denotes the covariant differentiations in the metric $\tilde{g}$ with respect to the local coordinates on $\Sigma\left(t_{0}\right)$.

Recall that by Lemma 3.2 we have

$$
\frac{\partial \tilde{g}_{i j}}{\partial t}=-2(F-\sigma) u \tilde{h}_{i j}
$$

On the other hand

$$
\begin{aligned}
\frac{\partial \tilde{g}_{i j}}{\partial t} & =2 e^{2 v} v_{t}\left(\sigma_{i j}+v_{i} v_{j}\right)+e^{2 v}\left(\dot{\sigma}_{i j}+\dot{v}_{i} v_{j}+v_{i} \dot{v}_{j}\right) \\
& =2 \tilde{g}_{i j} y w(F-\sigma)+e^{2 v}\left(\dot{\sigma}_{i j}+\dot{v}_{i} v_{j}+v_{i} \dot{v}_{j}\right) .
\end{aligned}
$$

Therefore at $\left(\mathbf{z}_{0}, t_{0}\right)$

$$
\dot{\sigma}_{i j}=-2 y(F-\sigma) \tilde{h}_{i j}-2 y(F-\sigma) \delta_{i j} .
$$

Combining equation (2.20) and (6.9) we get

$$
\begin{aligned}
\frac{\partial \tilde{h}_{i j}}{\partial t} & =\tilde{h}_{i j} y(F-\sigma)+\nabla_{i j}^{\prime}[y w(F-\sigma)]+2(F-\sigma) y \tilde{h}_{i j}+2 y(F-\sigma) \delta_{i j} \\
& =3 \tilde{h}_{i j} y(F-\sigma)+\nabla_{i j}^{\prime}[y w(F-\sigma)]+2 y(F-\sigma) \delta_{i j} \\
& =3 \tilde{h}_{i j} y(F-\sigma)+\left\{y \nabla_{i j}^{\prime} F+y(F-\sigma) v_{l i} v_{l j}\right. \\
& \left.-(F-\sigma) y \delta_{i j}+y_{i} F_{j}+y_{j} F_{i}\right\}+2 y(F-\sigma) \delta_{i j} \\
& =3 \tilde{h}_{i j} y(F-\sigma)+y \nabla_{i j}^{\prime} F+y(F-\sigma) v_{l i} v_{l j}+y_{i} F_{j}+y_{j} F_{i}+y(F-\sigma) \delta_{i j} .
\end{aligned}
$$

We can always assume at $\left(\mathbf{z}_{0}, t_{0}\right) \kappa_{\max }=g^{11} h_{11}$, thus we only need to compute $\dot{h}_{11}$ at this point. From now on, all calculations are done at $\left(\mathbf{z}_{0}, t_{0}\right)$ if no additional explanations.

Lemma 6.2. At $\left(\mathbf{z}_{0}, t_{0}\right)$,

$$
\begin{aligned}
\frac{\partial}{\partial t} h_{1}^{1}-y^{2} F^{i i} \nabla_{i i} h_{1}^{1} & =3(F-\sigma) \kappa_{1}^{2}+y^{2} F^{i j, k l} h_{i ; 1}^{j} h_{k ; 1}^{l} \\
& -(F-\sigma)+\left(\kappa_{1} \sum f_{i} \kappa_{i}^{2}+\kappa_{1} \sum f_{i}-F-\kappa_{1}^{2} F\right) .
\end{aligned}
$$


Proof. Differentiating equation (6.5) with respect to $t$ we get

$$
\dot{h}_{i j}=\frac{1}{u} \dot{\tilde{h}}_{i j}-\frac{\tilde{h}_{i j}}{u^{2}} \dot{u}+\frac{\nu^{n+1}}{u^{2}} \tilde{g}_{i j}+\frac{\nu^{n+1}}{u^{2}} \dot{\tilde{g}}_{i j}-2 \frac{\nu^{n+1}}{u^{3}} \tilde{g}_{i j} \dot{u} .
$$

Since

$$
\dot{u}=\frac{\partial e^{v} y}{\partial t}=e^{v} y v_{t}=y^{2}(F-\sigma)
$$

and

$$
\begin{aligned}
\dot{\nu}^{n+1} & =\frac{\partial}{\partial t}\left\{\frac{y-\nabla^{\prime} v \cdot \nabla^{\prime} y}{w}\right\} \\
& =-\nabla^{\prime}[y w(F-\sigma)] \cdot \nabla^{\prime} y \\
& =-(F-\sigma)\left(1-y^{2}\right)-y \nabla^{\prime} F \cdot \nabla^{\prime} y,
\end{aligned}
$$

we obtain

$$
\begin{aligned}
\dot{h}_{11} & =\frac{1}{y}\left[3 \tilde{h}_{11} y(F-\sigma)+y \nabla_{11}^{\prime} F+y(F-\sigma) v_{l 1} v_{l 1}+2 y_{1} F_{1}+y(F-\sigma)\right] \\
& -\tilde{h}_{11}(F-\sigma)-\frac{(F-\sigma)\left(1-y^{2}\right)}{y^{2}}-\frac{y \nabla^{\prime} F \cdot \nabla^{\prime} y}{y^{2}} \\
& -2(F-\sigma) \tilde{h}_{11}-2(F-\sigma) \\
& =\nabla_{11}^{\prime} F+(F-\sigma) v_{l 1}^{2}+\frac{2}{y} y_{1} F_{1}-\frac{(F-\sigma)}{y^{2}}-\frac{1}{y} \nabla^{\prime} F \cdot \nabla^{\prime} y \\
& =\nabla_{11}^{\prime} F+(F-\sigma)\left(\tilde{h}_{11}+1\right)^{2}+\frac{2}{y} y_{1} F_{1}-\frac{(F-\sigma)}{y^{2}}-\frac{1}{y} \nabla^{\prime} F \cdot \nabla^{\prime} y .
\end{aligned}
$$

Here we used $\tilde{h}_{i j}=v_{i j}-\delta_{i j}$ at $\left(\mathbf{z}_{0}, t_{0}\right)$.

By equation (2.1), (3.3) and (6.13) at the point $\left(\mathbf{z}_{0}, t_{0}\right)$ we get

$$
\dot{g}_{i j}=-2(F-\sigma) h_{i j} .
$$

On the other hand

hence

$$
\frac{\partial}{\partial t}\left(g^{i k} g_{i j}\right)=\frac{\partial}{\partial t} \delta_{j}^{k}=0
$$

$$
\dot{g}^{i k}=2 y^{4}(F-\sigma) h_{k i} .
$$

Finally we have

$$
\begin{aligned}
\frac{\partial h_{1}^{1}}{\partial t} & =\frac{\partial}{\partial t}\left(g^{1 k} h_{k 1}\right)=\dot{g}^{1 k} h_{k 1}+g^{1 k} \dot{h}_{k 1} \\
& =2 y^{4}(F-\sigma) h_{k 1}^{2}+y^{2} \dot{h}_{11} \\
& =2 y^{4}(F-\sigma) h_{1 k}^{2}+y^{2}\left[\nabla_{11}^{\prime} F+(F-\sigma)\left(\tilde{h}_{11}+1\right)^{2}+\frac{2}{y} y_{1} F_{1}\right. \\
& \left.-\frac{(F-\sigma)}{y^{2}}-\frac{1}{y} \nabla^{\prime} F \cdot \nabla^{\prime} y\right] .
\end{aligned}
$$

By (2.6) at $\left(\mathbf{z}_{0}, t_{0}\right)$ we get

$$
\nabla_{i j} f=\tilde{\nabla}_{i j} f+\frac{1}{y}\left(y_{i} f_{j}+y_{j} f_{i}-\sum y_{l} f_{l} \delta_{i j}\right) .
$$


Therefore we can rewrite equation (6.18) as

$$
\frac{\partial h_{1}^{1}}{\partial t}=3(F-\sigma) \kappa_{1}^{2}+y^{2} \nabla_{11} F-(F-\sigma) .
$$

Moreover

$$
\begin{aligned}
\nabla_{11} F & =F^{i i} h_{i ; 11}^{i}+F^{i j, k l} h_{i ; 1}^{j} h_{k ; 1}^{l} \\
& =F^{i i} \nabla_{11}\left(g^{i k} h_{k i}\right)+F^{i j, k l} h_{i ; 1}^{j} h_{k ; 1}^{l} \\
& =y^{2} F^{i i} \nabla_{11} h_{i i}+F^{i j, k l} h_{i ; 1}^{j} h_{k ; 1}^{l} .
\end{aligned}
$$

Thus

$$
\frac{\partial h_{1}^{1}}{\partial t}=3(F-\sigma) \kappa_{1}^{2}+y^{4} F^{i i} \nabla_{11} h_{i i}+y^{2} F^{i j, k l} h_{i ; 1}^{j} h_{k ; 1}^{l}-(F-\sigma) .
$$

Next let's recall the following well-known fundamental equations for a hypersurface $\Sigma$ in $\mathbb{H}^{n+1}$ :

Coddazzi equation: $\nabla_{i} h_{j k}=\nabla_{j} h_{k i}=\nabla_{k} h_{i j}$,

Gauss equation: $R_{i j k l}=\left(h_{i k} h_{j l}-h_{i l} h_{j k}\right)-\left(g_{i k} g_{j l}-g_{i l} g_{j k}\right)$,

Ricci equation: $\nabla_{l} \nabla_{k} h_{i j}-\nabla_{k} \nabla_{l} h_{i j}=h_{i p} g^{p q} R_{q j k l}+h_{j p} g^{p q} R_{q i k l}$.

So we have

$$
\begin{aligned}
\nabla_{11} h_{i i}-\nabla_{i i} h_{11} & =\nabla_{1} \nabla_{i} h_{1 i}-\nabla_{i} \nabla_{1} h_{1 i} \\
& =h_{1 p} g^{p q} R_{q i i 1}+h_{i p} g^{p q} R_{q 1 i 1} \\
& =\kappa_{1} R_{1 i i 1}+\kappa_{i} R_{i 1 i 1}
\end{aligned}
$$

and

$$
R_{1 i i 1}=-h_{11} h_{i i}+\frac{1}{y^{4}}, R_{i 1 i 1}=h_{i i} h_{11}-\frac{1}{y^{4}} .
$$

Substituting equation (6.24) into (6.23) and combining with equation (6.22) we obtain (6.11).

Lemma 6.3. At $\left(\mathrm{z}_{0}, t_{0}\right)$,

$$
\begin{aligned}
\dot{\nu}^{n+1}-y^{2} F^{i i} \nabla_{i i} \nu^{n+1} & =-(F-\sigma)\left(1-y^{2}\right)-\left(1-y^{2}\right) \sum f_{i}\left(\kappa_{i}-y\right) \\
& +y \sum f_{i}\left(\kappa_{i}-y^{2}\right)-2 y \sum f_{i} y_{i} \nu_{i}^{n+1} .
\end{aligned}
$$

Proof. By differentiating $\nu^{n+1}$ we get

$$
\begin{aligned}
\nu_{i}^{n+1} & =\left(\frac{y-\nabla^{\prime} v \cdot \nabla^{\prime} y}{w}\right)_{i} \\
& =\frac{y_{i}-v_{l i} y_{l}-v_{l} y_{l i}}{w}-\frac{y-\nabla^{\prime} v \cdot \nabla^{\prime} y}{w^{2}} w_{i} \\
& =y_{i}-v_{l i} y_{l}=-\tilde{h}_{i i} y_{i}
\end{aligned}
$$


and

$$
\begin{aligned}
\nu_{i j}^{n+1} & =\frac{y_{i j}-v_{l i j} y_{l}-v_{l i} y_{l j}-v_{l j} y_{l i}-v_{l} y_{l i j}}{w} \\
& -\frac{y-\nabla^{\prime} v \cdot \nabla^{\prime} y}{w^{2}} w_{i j}-w_{i}\left(\frac{y-\nabla^{\prime} v \cdot \nabla^{\prime} y}{w^{2}}\right)_{j} \\
& =-y \delta_{i j}-v_{l i j} y_{l}+y v_{l i} \delta_{l j}+y v_{l j} \delta_{l i}-y v_{l i} v_{l j} \\
& =-y_{l} \tilde{\nabla}_{l} \tilde{h}_{i j}-y \tilde{h}_{l i} \tilde{h}_{l j} .
\end{aligned}
$$

Then

$$
\begin{aligned}
\nabla_{i j} \nu^{n+1} & =\tilde{\nabla}_{i j} \nu^{n+1}+\frac{1}{y}\left(y_{i} \nu_{j}^{n+1}+y_{j} \nu_{i}^{n+1}\right)-\frac{1}{y} \sum y_{l} \nu_{l}^{n+1} \delta_{i j} \\
& =-y_{l} \nabla_{l} \tilde{h}_{i j}-y \tilde{h}_{l i} \tilde{h}_{l j}+\frac{1}{y}\left(y_{i} \nu_{j}^{n+1}+y_{j} \nu_{i}^{n+1}\right)-\frac{1}{y} \sum y_{l} \nu_{l}^{n+1} \delta_{i j} .
\end{aligned}
$$

Moreover, differentiating $F$ with respect to $\tau_{l}$,

$$
\begin{aligned}
\nabla_{l} F & =F^{i j}\left(h_{i}^{j}\right)_{l}=F^{i j}\left(u \tilde{h}_{i}^{j}+\nu^{n+1} \delta_{i j}\right)_{l} \\
& =F^{i j}\left\{y_{l} \tilde{h}_{i j}+y \nabla_{l} \tilde{h}_{i j}+\nu_{l}^{n+1} \delta_{i j}\right\} .
\end{aligned}
$$

Combining equations (6.14), 6.28) and (6.29) we have

$$
\begin{aligned}
& \dot{\nu}^{n+1}-y^{2} F^{i i} \nabla_{i i} \nu^{n+1} \\
& =-(F-\sigma)\left(1-y^{2}\right)-y\left(1-y^{2}\right) \sum f_{i}\left(\frac{\kappa_{i}}{y}-1\right)-y^{2} \sum F^{i i} y_{l} \tilde{\nabla}_{l} \tilde{h}_{i i}-y \sum \nu_{l}^{n+1} y_{l} \sum f_{i} \\
& +y^{2} \sum F^{i i} y_{l} \tilde{\nabla}_{l} \tilde{h}_{i i}+y^{3} \sum F^{i i} \tilde{h}_{i i}^{2}-2 y \sum F^{i i} y_{i} \nu_{i}^{n+1}+y \sum \nu_{l}^{n+1} y_{l} \sum f_{i} \\
& =-(F-\sigma)\left(1-y^{2}\right)-\left(1-y^{2}\right) \sum f_{i}\left(\kappa_{i}-y\right)+y \sum f_{i}\left(\kappa_{i}-y\right)^{2}-2 y \sum f_{i} y_{i} \nu_{i}^{n+1} .
\end{aligned}
$$

From the assumption

$$
\frac{\partial \varphi}{\partial t}-y^{2} F^{i i} \nabla_{i i} \varphi \geq 0 \text { at }\left(\mathbf{z}_{0}, t_{0}\right)
$$


we have that

$$
\begin{aligned}
0 & \leq \dot{h}_{1}^{1}-y^{2} F^{i i} \nabla_{i i} h_{1}^{1}-\frac{h_{1}^{1}}{\nu^{n+1}-a}\left(\dot{\nu}^{n+1}-y^{2} F^{i i} \nabla_{i i} \nu^{n+1}\right) \\
& =3(F-\sigma) \kappa_{1}^{2}+y^{2} F^{i j, k l} h_{i ; 1}^{j} h_{k ; 1}^{l}-(F-\sigma)+\kappa_{1} \sum f_{i} \kappa_{i}^{2}+\kappa_{1} \sum f_{i}-F-\kappa_{1}^{2} F \\
& -\frac{\kappa_{1}}{y-a}\left\{-(F-\sigma)\left(1-y^{2}\right)-\left(1-y^{2}\right) F+y\left(1-y^{2}\right) \sum f_{i}+y \sum f_{i} \kappa_{i}^{2}\right. \\
& \left.-2 y^{2} F+y^{3} \sum f_{i}-2 y \sum f_{i} y_{i} \nu_{i}^{n+1}\right\} \\
& =\left[3 \kappa_{1}^{2}-1+\frac{\kappa_{1}\left(1-y^{2}\right)}{y-a}\right](F-\sigma)+y^{2} F^{i j, k l} h_{i ; 1}^{j} h_{k ; 1}^{l} \\
& -\frac{a \kappa_{1}}{y-a}\left(\sum f_{i} \kappa_{i}^{2}+\sum f_{i}\right)+\left[-1-\kappa_{1}^{2}+\frac{\kappa_{1}\left(1+y^{2}\right)}{y-a}\right] F \\
& +\frac{2 y \kappa_{1}}{y-a} \sum f_{i} y_{i} \nu_{i}^{n+1} .
\end{aligned}
$$

Here we used lemma 6.2 and lemma 6.3. Since

$$
F^{i j, k l} h_{i ; 1}^{j} h_{k ; 1}^{l} \leq \sum_{i \neq j} \frac{f_{i}-f_{j}}{\kappa_{i}-\kappa_{j}}\left(h_{i ; 1}^{j}\right)^{2} \leq 2 \sum_{i \geq 2} \frac{f_{i}-f_{1}}{\kappa_{i}-\kappa_{1}}\left(h_{i ; 1}^{1}\right)^{2}
$$

and

$$
h_{i ; 1}^{1}=\nabla_{1}\left(g^{1 k} h_{k i}\right)=y^{2} h_{1 i ; 1}=y^{2} h_{11 ; i}=\frac{\kappa_{1}}{y-a} \nu_{i}^{n+1},
$$

we get

$$
F^{i j, k l} h_{i ; 1}^{j} h_{k ; 1}^{l} \leq \frac{2 \kappa_{1}^{2}}{(y-a)^{2}} \sum_{i \geq 2} \frac{f_{i}-f_{1}}{\kappa_{i}-\kappa_{1}}\left(\nu_{i}^{n+1}\right)^{2} .
$$

Therefore

$$
\begin{aligned}
& 0 \leq\left[3 \kappa_{1}^{2}-1+\frac{\kappa_{1}\left(1-y^{2}\right)}{y-a}\right](F-\sigma)+\frac{2 y^{2} \kappa_{1}^{2}}{(y-a)^{2}} \sum_{i \geq 2} \frac{f_{i}-f_{1}}{\kappa_{i}-\kappa_{1}}\left(\nu_{i}^{n+1}\right)^{2} \\
& -\frac{a \kappa_{1}}{y-a}\left(\sum f_{i} \kappa_{i}^{2}+\sum f_{i}\right)+\left[-1-\kappa_{1}^{2}+\frac{\kappa_{1}\left(1+y^{2}\right)}{y-a}\right] F+\frac{2 y \kappa_{1}}{y-a} \sum f_{i} y_{i} \nu_{i}^{n+1} .
\end{aligned}
$$

Let

$$
\begin{aligned}
& I=\left\{i: \kappa_{i}-y \leq-\theta \kappa_{1}\right\}, \\
& J=\left\{i:-\theta \kappa_{1}<\kappa_{i}-y<0, f_{i}<\theta^{-1} f_{1}\right\}, \\
& L=\left\{i:-\theta \kappa_{1}<\kappa_{i}-y<0, f_{i} \geq \theta^{-1} f_{1}\right\},
\end{aligned}
$$

where $\theta \in(0,1)$ is to be determined later. Then we have

$$
\begin{aligned}
\frac{-1}{y-a} \sum_{i \in I}\left(\kappa_{i}-\nu^{n+1}\right)^{2} f_{i} & \leq \frac{\theta \kappa_{1}}{y-a} \sum_{i \in I} f_{i}\left(\kappa_{i}-\nu^{n+1}\right) \\
& \leq \frac{\theta \kappa_{1}}{y-a} \sum_{i \in I} f_{i} y_{i}^{2}\left(\kappa_{i}-\nu^{n+1}\right)
\end{aligned}
$$


provided $\theta \kappa_{1} a>2$ we get

$$
\begin{aligned}
&-\frac{\kappa_{1} a}{y-a} \sum_{i \in I} f_{i}\left(\kappa_{i}-\nu^{n+1}\right)^{2} \leq+\frac{2 \kappa_{1}}{y-a} \sum_{i \in I} f_{i} y_{i}^{2}\left(\kappa_{i}-\nu^{n+1}\right) \\
&=-\frac{2 y \kappa_{1}}{y-a} \sum_{i \in I} f_{i} y_{i} \nu_{i}^{n+1} . \\
& \sum_{i \in J} f_{i} y_{i}^{2}\left(\nu^{n+1}-\kappa_{i}\right) \geq-\theta \kappa_{1} \sum_{i \in J} \theta^{-1} f_{1} y_{i}^{2} \geq f_{1} \kappa_{1},
\end{aligned}
$$

provided $a \kappa_{1}>2$,

$$
\frac{-a \kappa_{1}}{y-a} f_{1} \kappa_{1}^{2}+\frac{2 y \kappa_{1}}{y-a} \sum_{i \in J} f_{i} y_{i} \nu_{i}^{n+1}<0
$$

Finally, when $i \in L$,

$$
\begin{aligned}
& \frac{2 y \kappa_{1}}{y-a} \sum_{i \in L} f_{i} y_{i} \nu_{i}^{n+1}-\frac{2 y^{2} \kappa_{1}^{2}}{(y-a)^{2}} \sum_{i \in L} \frac{f_{i}-f_{1}}{\kappa_{1}-\kappa_{i}}\left(\nu_{i}^{n+1}\right)^{2} \\
& \leq \frac{-2 \kappa_{1}}{y-a}\left[\sum_{i \in L} f_{i} y_{i}^{2}\left(\kappa_{i}-\nu^{n+1}\right)+\frac{1}{y-a} \sum_{i \in L} \frac{1-\theta}{1+\theta} f_{i} y_{i}^{2}\left(\kappa_{i}-\nu^{n+1}\right)^{2}\right] \\
& =\frac{-2 \kappa_{1}}{y-a} \sum_{i \in L} f_{i} y_{i}^{2}\left[\left(\kappa_{i}-y\right)+\frac{1-\theta}{(y-a)(1+\theta)}\left(\kappa_{i}-y\right)^{2}\right] \\
& \leq \frac{\kappa_{1}(1+\theta)\left(1-y^{2}\right)}{2(1-\theta)} \sum_{i \in L} f_{i} .
\end{aligned}
$$

We want $\frac{\kappa_{1}(1+\theta)\left(1-y^{2}\right)}{2(1-\theta)}-\frac{a \kappa_{1}}{y-a} \leq 0$, which is equivalent to

$$
\phi_{\theta}(y)=a-\frac{(1+\theta)\left(1-y^{2}\right)(y-a)}{2(1-\theta)} \geq 0 \text { on } y \in(a, 1]
$$

Since

$$
\begin{aligned}
& \phi_{0}(y)=a-\frac{1}{2}\left(1-y^{2}\right)(y-a) \\
& >\frac{4}{3} a-\frac{1}{27} a^{3}-\frac{1}{27}\left(a^{2}+3\right)^{\frac{3}{2}}:=\phi(a) .
\end{aligned}
$$

For $a \in(0,1)$ it is easy to check that $\phi^{\prime}(a)>0, \phi(0)<0, \phi(1)>0$. Let $\sigma_{0}$ be the unique zero of $\phi(a)$ in $(0,1)$. Numerical calculation show that $0.14596<\sigma_{0}<$ 0.14597 .

\section{Convergence to a stationary solution}

Let us go back to our original equation (1.12), which is a scalar parabolic differential equation defined on the cylinder $\Omega_{T}=\Omega \times[0, T)$ with initial value $u(0)=u_{0} \in C^{\infty}(\Omega)$. In view of the a priori estimates, which we have estimated in the preceding sections, we know that

$$
\sqrt{1+|D u|^{2}} \leq \frac{1}{\sigma}
$$


and when $\sigma>\sigma_{0}\left(0.14596<\sigma_{0}<0.14597\right)$ there is a constant $C$ independent of $\epsilon$ and $t$ such that

$$
u\left|D^{2} u\right| \leq C .
$$

Thus we have

$$
F \text { is uniformly elliptic in } u \text {. }
$$

Moreover, since $F$ is concave, we have uniform $C^{2+\alpha}(\Omega)$ estimates for $u^{2}(t), \forall t>0$.

Therefore, the flow exists for all $t \geq 0$.

By integrating equation (1.12) with respect to $t$, we get

$$
u(x, t)-u(x, 0)=\int_{0}^{t}(F-\sigma) u w
$$

which implies

$$
\int_{0}^{\infty}(F-\sigma) u w<\infty \forall x \in \Omega
$$

Hence, for any $x \in \Omega$ there is a sequence $t_{k} \rightarrow \infty$ such that $(F-\sigma) u(x) \rightarrow 0$.

On the other hand, due to our assumptions on our initial surface, $u(x, \cdot)$ is monotone decreasing and therefore

$$
\lim _{t \rightarrow \infty} u(x, t)=\tilde{u}(x)
$$

exists, and is of class $C^{\infty}(\Omega)$. So $\tilde{u}(x)$ is a stationary solution of our problem.

\section{ACKNOWLEDGEMENT}

The author would like to thank Professor Joel Spruck for his guidance and support.

\section{REFERENCES}

[A94] B.Andrews, Contraction of convex hypersurfaces in Euclidean space, Calc.Var.PDE 2, (1994), 151-171.

[C89] Kung-Ching Chang, Heat flow and boundary value problem for harmonic maps, Annales de l'I. H. P., section C (1989), Vol 6, 363-395.

[CC95] L. Caffarelli, and X. Cabr, Fully nonlinear elliptic equations, American Mathematical Society,(1995).

[CNS84] L. Caffarelli, L. Nirenberg and J. Spruck, The Dirichlet problem for nonlinear secondorder elliptic equations I, Comm. Pure Applied Math. 37 (1984), 369-402.

[CNS85] L. Caffarelli, L. Nirenberg and J. Sprick, The Dirichlet problem for nonlinear secondorder elliptic equations III, Acta Math. 155 (1985), 261-301.

[CNS86] L. Caffarelli, L. Nirenberg and J. Sprick,Nonlinear second order elliptic equations IV, Current Topics in P.D.E. Kinokunize Co., (1986),1-26.

[G96] C.Gerhardt, Closed Weigngarten hypersurfaces in Riemannian manifolds, J.Differential Geom.43, (1996), 612-641.

[G06] C. Gerhardt, Curvature Problem, Int.Press, Somerville, MA, 2006.

[GS08] B. Guan, and J. Spruck, Hypersurfaces of constant curvature in Hyperbolic space II, preprint, arxiv.org/abs/0810.1781, J. Eur. Math. Soc. to appear.

[GS10] B.Guan and J.Spruck, Convex hypersurfaces of Constant curvature in hyperbolic space, preprint.

[GSZ09] B. Guan, J. Spruck and M. Szapiel, Hypersurfaces of constant curvature in Hyperbolic space I, J. Geom. Anal. 19 (2009), no. 4, 772-795. 
[H75] Richard S. Hamilton, Hamonic maps of manifolds with boundary, Lecture notes in mathematics 471, Springer (1975).

[ILT96] N. M. Ivochkina, M. Lin and N. S. Trudinger, The Dirichlet problem for the prescribed curvature quotient equations with general boundary values, Geometric Analysis and the Calculus of Variations, International Press, (1996), pp. 125-141.

[L96] Gray M. Lieberman, Second order parabolic differential equations, World Scientific Pub Co, (1996).

[LM97] Rafael Lopez and Sebastian Montiel, Existence of constant mean curvature graph in hyperbolic space, Calc. Var. Partial Differential Equations (1999), no. 2, 177-190.

[LX10] L. Lin and L. Xiao, Modified mean curvature flow of star-shaped hypersurfaces in hyperbolic space, preprint.

[NS96] B. Nelli, and J. Spruck, On the existence and uniqueness of constant mean curvature hypersurfaces in hyperbolic space, Geometric analysis and the calculus of variations, 253266, Int. Press, Cambridge, MA, (1996).

[LX11] L. Xiao, Curvature flow of complete convex hepersurfaces in hyperbolic space, preprint. 